\title{
Green's Hyperplane Restriction Theorem: an extension to modules
}

\author{
Ornella Greco
}

\begin{abstract}
In this paper, we prove a generalization of Green's Hyperplane Restriction Theorem to the case of modules over the polynomial ring, providing in particular an upper bound for the Hilbert function of the general linear restriction of a module $M$ in a degree $d$ by the corresponding Hilbert function of a lexicographic module.
\end{abstract}

\section{Introduction}

The extremal properties of the Hilbert function constitute a relevant topic in commutative algebra and, within this topic, an important role is played by lexicographic ideals and binomial representation of integers.

Many results about extremal behavior of Hilbert functions have been proved. In particular, Macaulay's Theorem (see [3, 10, 13]), which characterizes the possible Hilbert functions of homogeneous $k$-algebras; or, the analogous result for exterior algebra, proved by Kruskal and Katona (see [9, 12]), which also provides a characterization of $f$-vectors of simplicial complexes.

More recently, in [8], Hulett proved an extension of Macaulay's Theorem to modules over the symmetric algebra.

Another classical, but slightly recent, result in this topic is the Hyperplane Restriction Theorem (HRT), given by Green in [7], which gives a bound for the codimension of the generic linear restriction of a vector space generated in a certain degree by the codimension of a lex-segment space with same degree and dimension. This result was also used by Green to give another, less technical, proof of Macaulay's Theorem.

It has been also been applied by several authors (see for instance [1, 2, 14]) to get some results about level and Gorenstein algebras, with focus on the 
weak Lefschetz property.

In this paper, we will focus on Green's theorem, trying to follow Hulett's path in generalizing these results to modules over the polynomial ring. One generalization in this direction has already been done by Gasharov ([5]), but we will give an extension of Green's theorem that provides a bound which is actually achieved by lexicographic modules.

We will first give the tools to state Green's Hyperplane Restriction Theo-

rem: namely we introduce the binomial expansion of a positive integer, also called Macaulay representation, then, the concept of generic linear forms. Afterwards, we will state Green's theorem.

In the next section, we will extend some of these definitions to the submodule of a finitely generated free module, in particular we will define a monomial order induced by the deglex ordering, and will provide the concept of lexicographic module, which is a particular class of monomial modules.

We first try to extend Green's theorem in the simplest case of a rank 2 module, by proving an inequality of Macaulay representations. Later we prove by induction a new inequality which imply the main theorem, namely the extension of the Hyperplane Restriction Theorem to the case of modules over the polynomial ring.

As a consequence of our theorem, we will derive a result which constitute another version of the theorem "after scaling", which means that we divide by the respective Hilbert function of the polynomial ring.

Finally, we apply our main theorem to level algebras, by giving some conditions in which the bound given by the extension to modules of the Green's HRT is given than the one obtained trough the Green's HRT.

\section{Green's Theorem}

Let $S=k\left[x_{1}, \ldots, x_{n}\right]$, where $k$ is an infinite field, and let $S$ be standard graded. Let us fix the deglex monomial ordering on $S$ with $x_{1}>x_{2}>\cdots>$ $x_{n}$, namely

$$
\mathbf{x}^{\mathbf{a}}>_{\text {deglex }} \mathbf{x}^{\mathbf{b}} \Leftrightarrow|\mathbf{a}|>|\mathbf{b}| \text { or }|\mathbf{a}|=|\mathbf{b}| \text { and } \mathbf{a}>_{\text {lex }} \mathbf{b}
$$

(see [11]). Notice that in a homogeneous component $S_{d}$ the monomial orderings, deglex and lex, coincide. 
Definition 2.1. Let $a, d \in \mathbb{N}$, then the $d$-th Macaulay representation of a (also called the binomial representation of $a$ in base $d$ ) is the unique way to write

$$
a=\left(\begin{array}{c}
a_{d} \\
d
\end{array}\right)+\left(\begin{array}{c}
a_{d-1} \\
d-1
\end{array}\right)+\cdots+\left(\begin{array}{c}
a_{\delta} \\
\delta
\end{array}\right)
$$

where $a_{d}>a_{d-1}>\cdots>a_{\delta} \geq \delta$ and $\delta=\min \left\{i \mid a_{i} \geq i\right\}$.

Let us set $\left(\begin{array}{l}c \\ d\end{array}\right)=0$ whenever $c<d$, then, given the $d$-th Macaulay representation of $a$, let us define the integer

$$
a_{\langle d\rangle}=\left(\begin{array}{c}
a_{d}-1 \\
d
\end{array}\right)+\left(\begin{array}{c}
a_{d-1}-1 \\
d-1
\end{array}\right)+\cdots+\left(\begin{array}{c}
a_{\delta}-1 \\
\delta
\end{array}\right) .
$$

Sometimes it will be convenient to use the extended $d$-th Macaulay representation, i.e. $\left(\begin{array}{c}a_{d} \\ d\end{array}\right)+\left(\begin{array}{c}a_{d-1} \\ d-1\end{array}\right)+\cdots+\left(\begin{array}{c}a_{1} \\ 1\end{array}\right)$, where $a_{d}>a_{d-1}>\cdots>a_{1} \geq 0$.

Moreover, since $\left(\begin{array}{l}a \\ b\end{array}\right)=0$ if $a<b$, we will set the numerators of such binomial coefficients in the Macaulay representation equal to zero.

Observation 2.2. If $a=\left(\begin{array}{c}a_{d} \\ d\end{array}\right)+\left(\begin{array}{c}a_{d-1} \\ d-1\end{array}\right)+\cdots+\left(\begin{array}{c}a_{1} \\ 1\end{array}\right)$ and $b=\left(\begin{array}{c}b_{d} \\ d\end{array}\right)+\left(\begin{array}{c}b_{d-1} \\ d-1\end{array}\right)+\cdots+\left(\begin{array}{c}b_{1} \\ 1\end{array}\right)$, $a \geq b$ if and only if $\left(a_{d}, \ldots, a_{1}\right) \geq_{l e x}\left(b_{d}, \ldots, b_{1}\right)$.

Definition 2.3. Let $d \in \mathbb{N}$, a lex-segment is a set constituted by all monomials in $S_{d}$ lexicographically larger or equal than $f$, for some $f \in \operatorname{Mon}\left(S_{d}\right)$. A $k$-vector subspace $V$ of $S_{d}$ is a lex-segment space if $\operatorname{Mon}\left(S_{d}\right) \cap V$ is a $k$-basis of $V$ and a lex-segment.

Definition 2.4. A graded monomial ideal $I$ in $S$ is said to be lexicographic if, for all degrees $d$, the homogeneous component $I_{d}$ is a lex-segment space.

Definition 2.5. We say that a property $\mathcal{P}$ holds for a generic linear form $\ell$ if there is a non-empty Zariski open set $\mathcal{U} \subseteq S_{1}$ such that $\mathcal{P}$ holds for all $\ell \in \mathcal{U}$.

If $V \subseteq S_{d}$ a lex-segment space, we denote by $V_{\ell}$ the image of $V$ in $S /(\ell)$.

Proposition 2.6. [10, Proposition 5.5.23] Let $k$ be an infinite field, $d \in \mathbb{N}$, $V \subseteq S_{d}$ a lex-segment space. Then:

1. For a generic linear form $\ell \in S_{1}$, there is a homogeneous linear change of coordinates $\phi: S \rightarrow S$ such that $\phi(V)=V$ and $\phi(\ell)=x_{n}$;

2. For a generic linear form $\ell \in S_{1}$, we have

$$
\operatorname{codim}_{k}\left(V_{\ell}\right)=\operatorname{codim}_{k}\left(V_{x_{n}}\right)=\operatorname{codim}_{k}(V)\langle d\rangle .
$$


So, after a general change of coordinates, we may assume that the generic linear form is $x_{n}$.

From now on, we will denote by $H(M, d)$ the Hilbert function of the $S$ module $M$ in degree $d$, i.e. $\operatorname{dim}_{k}\left(M_{d}\right)$. Moreover, if $\ell \in S_{1}$ is a linear form and $R=S / I$, we denote by $R_{\ell}$ the ring $S /[I+(\ell)]$.

Theorem 2.7 (Green's Hyperplane Restriction Theorem). Let I be a homogeneous ideal in $S, d \in \mathbb{N}$, then

$$
H\left((S / I)_{\ell}, d\right) \leq H(S / I, d)_{\langle d\rangle},
$$

where $\ell$ is a generic linear form. Moreover, equality holds when $I_{d}$ is a lexsegment space.

\section{Extension to modules}

In the case of the restriction of an $S$-module $M$ to a generic hyperplane $\ell$ we expect not only an upper bound on the Hilbert function $H\left(M_{\ell}, d\right)$, but also that this upper bound is fully described by lexicographic modules, in complete analogy with Green's theorem.

In this section, we will be able to prove some numerical properties of the Macaulay's representations, that will lead to a version of Green's HRT for modules valid in any characteristic of the infinite field $k$.

Let $F$ be a finitely generated graded free $S$-module, let us fix a homogeneous basis $\left\{e_{1}, e_{2}, \ldots, e_{r}\right\}$ and let $\operatorname{deg}\left(e_{i}\right)=f_{i}$, where, without loss of generality, we may assume that and $f_{1} \leq f_{2} \leq \cdots \leq f_{r}$.

We now define the monomial modules and we induce a monomial ordering on $F$ in such a way that the concept of lexicographic module may be defined. We also define another monomial ordering on $F$, a particular revlex, that will let us use the tool of generic initial modules in our circumstances.

Definition 3.1. A monomial in $F$ is an element of the form $m e_{i}$ where $m \in \operatorname{Mon}(S)$. A submodule $M \subseteq F$ is monomial if it is generated by monomials, in this case it can be written as $I_{1} e_{1} \oplus I_{2} e_{2} \oplus \cdots \oplus I_{r} e_{r}$, where $I_{i}$ is a monomial ideal for $i=1,2, \ldots, r$. 
One can extend a monomial ordering, defined in the polynomial ring $S$, to a finitely generated free $S$-module in a really natural way, in particular here we define the deglex ordering in $F$.

Definition 3.2. Given two monomials in $F, m e_{i}$ and $n e_{j}$, we say that $m e_{i}>_{\text {deglex }} n e_{j}$ if either $i=j$ and $m>_{\text {deglex }} n$ in $S$ or $i<j$. In particular, we have that $e_{1}>e_{2}>\cdots>e_{r}$.

Again, as in the polynomial ring, when we restrict to a precise homogeneous component, the two induced monomial orderings, deglex and lex, coincide.

Definition 3.3. A monomial graded submodule $L$ is a lexicographic module if, for every degree $d, L_{d}$ is spanned by the largest, with respect to the lexicographic order (or deglex), $H(L, d)$ monomials.

Let us now recall the definition of reverse lexicographic order on $F$ as given in ([4, pg. 339]), some easy results about it.

Definition 3.4. The reverse lexicographic order on $F$ is defined by choosing an order on the basis of $F$, say $e_{1}>\cdots>e_{r}$ and by setting $m e_{i}>_{\text {revlex }} n e_{j}$ iff either $\operatorname{deg}\left(m e_{i}\right)>\operatorname{deg}\left(n e_{j}\right)$ or the degrees are the same and $m>_{\text {revlex }} n$ in $S$ or $m=n$ and $i<j$.

Remark 3.5. Notice that the deglex is a POT (Position over Term) monomial order, on the contrary the revlex is TOP (Term over Position) monomial order.

Definition 3.6. The initial module of a submodule $M$, denoted by $\operatorname{in}(M)$, is the submodule of $F$ generated by the set $\{\operatorname{in}(m) \mid m \in M\}$, i.e. by all the leading terms (according to the lexicographic order or deglex or revlex) of elements in $M$.

Proposition 3.7. [4, Proposition 15.12] Suppose that F is a free S-module with basis $\left\{e_{1}, \ldots, e_{r}\right\}$ and reverse lexicographic order. Let $M$ be a graded submodule.

Then $\operatorname{in}\left(M+x_{n} F\right)=\operatorname{in}(M)+x_{n} F$ and $\left(\operatorname{in}(M):_{F} x_{n}\right)=\operatorname{in}\left(M:_{F} x_{n}\right)$.

Let us define the concept of generic initial module as done in ([15, Chapters 1 and 2]).

Let $\operatorname{GL}(n)$ be the general linear group. It acts on $S$ by $\left(a_{i j}\right) x_{j}=\sum_{i=1}^{n} a_{i j} x_{i}$. 
Let $\mathrm{GL}(F)$ be the group of the $S$-module automorphisms of $F$. An element $\phi$ in $\operatorname{GL}(F)$ is a homogeneous automorphism and can be represented by a matrix $\left(t_{i j}\right)$, with $t_{i j} \in S_{f_{i}-f_{j}}$, where $\phi\left(e_{i}\right)=\sum_{j=1}^{r} t_{i j} e_{j}$. We also have an action of $\operatorname{GL}(n)$ on $\operatorname{GL}(F)$ given by $a \cdot \phi=a \phi a^{-1}$, for all $a \in \operatorname{GL}(n)$ and $\phi \in \mathrm{GL}(F)$. The two groups together act on $F$ by mean of their semi-direct product $G=\mathrm{GL}(n) \rtimes \mathrm{GL}(F)$.

Let us consider $B(n)$ the subgroup of $\mathrm{GL}(n)$ of upper triangular invertible matrices: from the action given above, a matrix in $B(n)$ will send $x_{1}$ to a multiple of itself, and $x_{n}$ to the linear combination of all variables $x_{i}$ 's with coefficients the last column of tha matrix. Let $B(F)$ be the subgroup of all automorphisms in $\mathrm{GL}(F)$ represented by lower triangular matrices: they send each $e_{l}$ to an $S$-linear combination of $e_{1}, \ldots, e_{l}$. Let $B=B(n) \rtimes B(F)$. Pardue, in [15], proved the generalization to modules of the Galligo's theorem, in a more general setting than ours, and in particular he proved the following result:

Proposition 3.8. Let $M \subset F$ be a graded module, and let $<$ be a monomial order on $F$, then there exists a Zariski open set $U \subseteq G$ such that $\operatorname{in}_{<}(\phi M)$ is constant for every $\phi \in U$. Moreover, $\mathrm{in}_{<}(\phi \mathrm{M})$ is fixed by the action of group $B$.

Definition 3.9. The monomial submodule $\operatorname{in}_{<}(\phi M), \phi \in U$, is called the generic initial module of $M$, and denoted by $\operatorname{gin}_{<}(\mathrm{M})$.

The generic initial module has really nice properties in case we use, as monomial order on $F$, the revlex order defined above.

Proposition 3.10. For a generic linear form $\ell$ and a graded submodule $M$ of $F$, we have that

$$
\operatorname{gin}\left(\mathrm{M}_{\ell}\right)=\operatorname{gin}(\mathrm{M})_{x_{n}} .
$$

The revlex gin(M) has the properties in 3.7, and moreover, since it is B-fixed, we can work in generic coordinates, and choose the coordinates in such a way that $\ell=x_{n}$.

If $\ell \in S_{1}$ is a linear form, and $M$ a graded submodule of $F$, let us denote by $(F / M)_{\ell}$ the restriction of the module $F / M$ to $\ell$, which is equal to $F /(M+\ell F)$. Notice that $F_{\ell}$ is a module over $S_{\ell}$ and that $S_{\ell}$ can be thought as polynomial ring in $n-1$ variables. 
Before the statement of the main theorem of the section, we are going to prove two propositions, regarding the behavior of the function $*\langle d\rangle: \mathbb{N} \rightarrow \mathbb{N}$ : in particular we need to provide an upper bound to the function $a_{\left\langle d_{1}\right\rangle}+b_{\left\langle d_{2}\right\rangle}$, where $d_{1} \geq d_{2}$, generalizing [5, Lemma 4.4]; and then, we will extend the latter inequality to the sum of $r=\operatorname{rank}(F)$ integers.

These two proposition will be just the numerical translations of the extension of Green's theorem, respectively in the $\operatorname{rank}(F)=2$ case, and in the $\operatorname{rank}(F)=r$ case.

Lemma 3.11. [5, Lemma 4.4, Lemma 4.5] For any $a, b, d \in \mathbb{N}$, the two following inequalities hold: $a_{\langle d\rangle}+b_{\langle d\rangle} \leq(a+b)_{\langle d\rangle} ; a_{\langle d+1\rangle} \leq a_{\langle d\rangle}$.

Remark 3.12. If $a, d \in \mathbb{N}$ and $a=\left(\begin{array}{c}a_{d} \\ d\end{array}\right)+\cdots+\left(\begin{array}{c}a_{\delta} \\ \delta\end{array}\right)\left(a_{\delta} \geq \delta\right)$ is the $d$-th Macaulay representation of $a$, then

$$
(a-1)_{\langle d\rangle}=a_{\langle d\rangle} \text { if and only if } a_{\delta}=\delta .
$$

Proposition 3.13. Given $a, b \in \mathbb{N}, a \leq N_{1}=\left(\begin{array}{c}n+d_{1}-1 \\ d_{1}\end{array}\right), b \leq N_{2}=\left(\begin{array}{c}n+d_{2}-1 \\ d_{2}\end{array}\right)$ and $d_{1} \geq d_{2}, d_{1}, d_{2} \in \mathbb{N}$, then

$$
a_{\left\langle d_{1}\right\rangle}+b_{\left\langle d_{2}\right\rangle} \leq \begin{cases}(a+b)_{\left\langle d_{2}\right\rangle} & \text { if } a+b \leq N_{2}, \\ \left(a+b-N_{2}\right)_{\left\langle d_{1}\right\rangle}+\left(N_{2}\right)_{\left\langle d_{2}\right\rangle} & \text { if } a+b \geq N_{2} .\end{cases}
$$

Proof We will now prove the claim by induction on $i=a+b$.

The case $i=0$ is trivial. Thus, let the claim be true for all $a^{\prime}, b^{\prime}$ such that $0<a^{\prime}+b^{\prime}<i$.

Let $a=\left(\begin{array}{c}a_{d_{1}} \\ d_{1}\end{array}\right)+\cdots+\left(\begin{array}{c}a_{d_{2}} \\ d_{2}\end{array}\right)+\cdots+\left(\begin{array}{c}a_{1} \\ 1\end{array}\right), b=\left(\begin{array}{c}b_{d_{2}} \\ d_{2}\end{array}\right)+\cdots+\left(\begin{array}{c}b_{1} \\ 1\end{array}\right)$, where some of the last top coefficients in the two Macaulay representations may be zero, and let us denote these Macaulay representations as vectors $a=\left(a_{d_{1}}, \ldots, a_{1}\right)$ and $b=\left(b_{d_{2}}, \ldots, b_{1}\right)$.

Let us now distinguish between two cases.

First Case If the $d_{1}$-Macaulay representation of $a$ ends after degree $d_{2}$, i.e. $\min \left\{i \mid a_{i} \geq i\right\} \leq d_{2}$, we write $a=\left(a_{d_{1}}, \ldots, a_{d_{2}}, \ldots, a_{1}\right)$ and $b=\left(b_{d_{2}}, \ldots, b_{1}\right)$, where in both vectors the last entries, after degree $d_{2}$, may be zero.

Then, we redefine $a$ and $b$ as follows, sorting them entry by entry:

$$
\begin{aligned}
& a=\left(a_{d_{1}}, \ldots, a_{d_{2}+1}, \min \left\{a_{d_{2}}, b_{d_{2}}\right\}, \ldots, \min \left\{a_{1}, b_{1}\right\}\right), \\
& b=\left(\max \left\{a_{d_{2}}, b_{d_{2}}\right\}, \ldots, \max \left\{a_{1}, b_{1}\right\}\right) .
\end{aligned}
$$


Observe that, in this process, we are decreasing $a$ of the same amount we increase $b$ (we are not modifying $a+b$ ) and we are not changing $a_{\left\langle d_{1}\right\rangle}+b_{\left\langle d_{2}\right\rangle}$, since the sum of the two binomial expansions will still contain the same terms. Moreover, in this way, we obtain that the Macaulay representation of $a$ ends sooner than that of $b$.

The idea is to find $a^{\prime}, b^{\prime}$ such that $a^{\prime}+b^{\prime}=a+b$ and $a \leq a^{\prime}, b^{\prime} \geq b$ and such that the following are satisfied:

1. $a_{\left\langle d_{1}\right\rangle}^{\prime}+b_{\left\langle d_{2}\right\rangle}^{\prime} \geq a_{\left\langle d_{1}\right\rangle}+b_{\left\langle d_{2}\right\rangle}$;

2. if $i<N_{2}$, either $a^{\prime}=0$ and $b^{\prime}=i$; if $i \geq N_{2}$, either $a^{\prime}=i-N_{2}$ and $b^{\prime}=N_{2}$; or at least one between $a^{\prime}$ and $b^{\prime}$ has Macaulay representation ending with a binomial coefficient of the form $\left(\begin{array}{l}t \\ t\end{array}\right)$ (since, in this case, we can use Remark 3.12 and apply induction hypothesis).

So, we can suppose that

$$
\begin{aligned}
a & =\left(a_{d_{1}}, \ldots, a_{d_{2}}, a_{d_{2}-1}, \ldots, a_{s}, 0, \ldots, 0\right) \\
b & =\left(b_{d_{2}}, b_{d_{2}-1}, \ldots, b_{t}, 0, \ldots, 0\right)
\end{aligned}
$$

where $a_{s}>s$ and $b_{t}>t$ (otherwise we can use Remark 3.12 and apply induction hypothesis), and $s \geq t$.

We need to show how to find $a^{\prime}$ and $b^{\prime}$, by decreasing $a$ and increasing $b$ of the same amount in three different cases.

In the case in which $s=t=1$, we have $a_{1}>1$ and $b_{1}>1$, we can consider $\left(a_{d_{1}}, \ldots, a_{d_{2}}, \ldots, a_{1}-1\right)$ and $\left(b_{d_{2}}, \ldots, b_{2}, b_{1}+1\right)$, without changing $a_{\left\langle d_{1}\right\rangle}+b_{\left\langle d_{2}\right\rangle}$. Applying this several times we will get to the point in which $a_{1}=1$, so we have reached the desired $a^{\prime}$ and $b^{\prime}$.

The second case is when $t=1$ and $s>1$, here we have to write $\left(\begin{array}{c}a_{s} \\ s\end{array}\right)=\sum_{j=0}^{s}\left(\begin{array}{c}a_{s}-(j+1) \\ s-j\end{array}\right)$, and then consider $\left(a_{d_{1}}, \ldots, a_{d_{2}}, \ldots, a_{s+1}, a_{s}-\right.$ $\left.1, \ldots, a_{s}-s\right)$ and $\left(b_{d}, \ldots, b_{2}, b_{1}+1\right)$ (we are decreasing $a$ and increasing $b$ by 1$)$. After this step, we are again in the first case.

Notice that in these first two cases if $b_{1}+1=b_{2}$ we have to consider $\left(b_{d_{2}}, \ldots, b_{2}+1,0\right)$ and then we sort again by taking the $\min \left\{a_{i}, b_{i}\right\}$ and the $\max \left\{a_{i}, b_{i}\right\}$.

The last case is when $s>t>1$ : here we write $\left(\begin{array}{c}a_{s} \\ s\end{array}\right)=\sum_{j=0}^{s-t}\left(\begin{array}{c}a_{s}-(j+1) \\ s-j\end{array}\right)+$ 
$\left(\begin{array}{c}a_{s}-(s-t+1) \\ t-1\end{array}\right)$; so we consider:

$$
\begin{aligned}
& \left(a_{d_{1}}, \ldots, a_{d_{2}}, \ldots, a_{s+1}, a_{s}-1, \ldots, a_{s}-(s-t+1)(\text { position } t),\right. \\
& \left.a_{s}-(s-t+1)(\text { position } t-1), 0, \ldots, 0\right) \\
& \left(b_{d_{2}}, b_{d_{2}-1}, \ldots, b_{t}, 0, \ldots, 0\right),
\end{aligned}
$$

and we resort by taking the min and the max in each modified degree.

Second Case When the Macaulay representation of $a$ ends before the degree $d_{2}$, say in degree $j$, say $k$ is the smallest degree for which $b_{k} \geq k$. If $d_{2}>1$, then let us consider the decomposition:

$$
\left(\begin{array}{c}
a_{j} \\
j
\end{array}\right)=\sum_{i=k+1}^{j}\left(\begin{array}{c}
a_{j}-(j-i+1) \\
i
\end{array}\right)+\left(\begin{array}{c}
a_{j}-(j-k) \\
k
\end{array}\right) ;
$$

so we write

$$
\begin{aligned}
a= & \left(a_{d_{1}}, \ldots, a_{j+1}, a_{j}-1, \ldots, a_{j}-\left(j-d_{2}+1\right), \ldots,\right. \\
& \left.a_{j}-(j-k), a_{j}-(j-k), 0, \ldots, 0\right),
\end{aligned}
$$

and we compare, entry by entry, with $b$ moving the smaller entries in the new vector $a$ and the larger entries in $b$.

The only case in which this cannot work is in the trivial case $b=(\beta)$, $a=\left(a_{d_{1}}, \ldots, \alpha, \alpha\right)$ (after having used the previous decomposition) and $\alpha=\beta$, in this situation we just consider $\left(a_{d_{1}}, \ldots, \alpha, \alpha-1\right)$ and $(\beta+1)$.

So, for all the possible cases we have found a way to subtract to $a$ and add to $b$ the same number in such a way that the function $f(x, y)=x_{\left\langle d_{1}\right\rangle}+y_{\left\langle d_{2}\right\rangle}$ does not change: we will continue, recursively, to do this until we get the extremal cases, or until one of the two numbers has Macaulay representation ending with $\left(\begin{array}{l}t \\ t\end{array}\right)$.

Observation 3.14. Notice that the previous proposition is just the numerical translation of the generalization of Green's HRT to the case in which we have $F=S e_{1} \oplus S e_{2}$, with $e_{1}>_{\text {deglex }} e_{2}, \operatorname{deg}\left(e_{1}\right)=f_{1}, \operatorname{deg}\left(e_{2}\right)=f_{2}$ and $f_{1} \leq f_{2}$. In fact, for any degree $d \in \mathbb{N}$, we set $d_{1}=d-f_{1}, d_{2}=d-f_{2}$, and $N_{1}=\left(\begin{array}{c}n+d_{1}-1 \\ d_{1}\end{array}\right)=\operatorname{dim}_{k}\left(S_{d_{1}}\right), N_{2}=\left(\begin{array}{c}n+d_{2}-1 \\ d_{2}\end{array}\right)=\operatorname{dim}_{k}\left(S_{d_{2}}\right)$. 
Remark 3.15. The previous proposition implies the first part of Lemma 3.11: in fact, when we have $f_{1}=f_{2}$, and so $N_{1}=N_{2}=N$, we can repeat the same argument of the proof of Proposition 3.13 , with the only difference that we continue decreasing $a$ and increasing $b$ of the same amount until $a=0$ and $b=i$, i.e. without distinguish between the cases in which $i \leq N$ and $i \geq N$.

The following proposition just extends the previous inequality to the case of more summands.

Proposition 3.16. Let $d_{1}, \ldots, d_{r} \in \mathbb{N}$ a non-increasing sequence, and set $N_{i}=\left(\begin{array}{c}n+d_{i}-1 \\ d_{i}\end{array}\right)$. Let $a_{i} \leq N_{i}$ be an integer. Then, if, for some $j, \sum_{i=j+1}^{r} N_{i} \leq$ $\sum_{i=1}^{r} a_{i} \leq \sum_{i=j}^{r} N_{i}$, the following inequality holds:

$$
\sum_{i=1}^{r} a_{i\left\langle d_{i}\right\rangle} \leq\left(\sum_{i=1}^{r} a_{i}-\sum_{i=j+1}^{r} N_{i}\right)_{\left\langle d_{j}\right\rangle}+\sum_{i=j+1}^{r} N_{i\left\langle d_{i}\right\rangle}
$$

Proof By induction on $r$. The case $r=2$ is given by the previous result, let us now suppose that the inequality holds for $s<r$. Let $\sum_{i=j+1}^{r} N_{i} \leq$ $\sum_{i=1}^{r} a_{i} \leq \sum_{i=j}^{r} N_{i}$. We then distinguish between two cases.

In case $a_{j}+a_{j+1} \leq N_{j+1}$, then $a_{j\left\langle d_{j}\right\rangle}+a_{j+1}\left\langle d_{j+1}\right\rangle \leq\left(a_{j}+a_{j+1}\right)_{\left\langle d_{j+1}\right\rangle}$.

Since $\sum_{i=j+1}^{r} N_{i} \leq \sum_{i=1}^{r} a_{i} \leq \sum_{i=j}^{r} N_{i} \leq N_{j-1}+\sum_{i=j+1}^{r} N_{i}$, we can apply the induction to have the following:

$$
\begin{aligned}
& \sum_{i=1}^{r} a_{i\left\langle d_{i}\right\rangle} \leq \sum_{i=1, i \neq j, j+1}^{r} a_{i\left\langle d_{i}\right\rangle}+\left(a_{j}+a_{j+1}\right)_{\left\langle d_{j+1}\right\rangle} \leq\left(\sum_{i=1}^{r} a_{i}-\sum_{i=j+1}^{r} N_{i}\right)_{\left\langle d_{j-1}\right\rangle}+ \\
& +\sum_{i=j+1}^{r} N_{i\left\langle d_{i}\right\rangle} \leq\left(\sum_{i=1}^{r} a_{i}-\sum_{i=j+1}^{r} N_{i}\right)_{\left\langle d_{j}\right\rangle}+\sum_{i=j+1}^{r} N_{i\left\langle d_{i}\right\rangle} .
\end{aligned}
$$

In the case in which $a_{j}+a_{j+1} \geq N_{j+1}$, then, by the base of induction, $a_{j\left\langle d_{j}\right\rangle}+a_{j+1}\left\langle d_{j+1}\right\rangle \leq\left(a_{j}+a_{j+1}-N_{j+1}\right)_{\left\langle d_{j}\right\rangle}+N_{j+1}\left\langle d_{j+1}\right\rangle$. We have that $a_{j}+$ $a_{j+1}-N_{j+1} \leq N_{j}$ and $\sum_{i=j+2}^{r} N_{i} \leq \sum_{i=1}^{r} a_{i}-N_{j+1} \leq N_{j}+\sum_{i=j+2}^{r} N_{i}$, thus by induction:

$$
\sum_{i=1, i \neq j, j+1}^{r} a_{i\left\langle d_{i}\right\rangle}+\left(a_{j}+a_{j+1}-N_{j+1}\right)_{\left\langle d_{j}\right\rangle} \leq\left(\sum_{i=1}^{r} a_{i}-\sum_{i=j+1}^{r} N_{i}\right)_{\left\langle d_{j}\right\rangle}+\sum_{i=j+2}^{r} N_{i\left\langle d_{i}\right\rangle}
$$


By adding $N_{j+1}\left\langle d_{j+1}\right\rangle$ to both sides, we get

$$
\sum_{i=1}^{r} a_{i\left\langle d_{i}\right\rangle} \leq\left(\sum_{i=1}^{r} a_{i}-\sum_{i=j+1}^{r} N_{i}\right)_{\left\langle d_{j}\right\rangle}+\sum_{i=j+1}^{r} N_{i\left\langle d_{i}\right\rangle}
$$

The idea for the extension of the Green's theorem to modules is to reduce to the monomial case: a monomial submodule is direct sum of monomial components, and so we may just apply Theorem 2.7 to each of these components. Afterwards we need to bound the Hilbert function of the quotient by the monomial submodule by the Hilbert function of the quotient by lexicographic module.

Let us now give a definition that will be used inside the statement of our generalization of Green's theorem, and then we are ready to prove our main result.

Definition 3.17. Let $M$ be a submodule in $F$, and $m \in \mathbb{N}$. Set $d_{i}=m-f_{i}$ $\left(\left\{d_{1}, d_{2}, \ldots, d_{r}\right\}\right.$ is a non-increasing sequence) and $N_{i}=\left(\begin{array}{c}n+d_{i}-1 \\ d_{i}\end{array}\right)=\operatorname{dim}_{k} S_{d_{i}}$. Then, if $\sum_{i=j+1}^{r} N_{i} \leq H(F / M, m) \leq \sum_{i=j}^{r} N_{i}$, for some $j$, we define

$$
H(F / M, m)_{\{m, r\}}=\left(H(F / M, m)-\sum_{i=j+1}^{r} N_{i}\right)_{\left\langle d_{j}\right\rangle}+\sum_{i=j+1}^{r} N_{i\left\langle d_{i}\right\rangle} .
$$

Theorem 3.18. Let $F=S e_{1} \oplus \cdots \oplus S e_{r}$ where $\operatorname{deg}\left(e_{i}\right)=f_{i}$ for all $i$. Let $M$ be a submodule in $F$, then

$$
H\left((F / M)_{\ell}, m\right) \leq H\left((F / L)_{\ell}, m\right)
$$

where $\ell$ is generic linear form, $m \in \mathbb{N}$, and $L$ is a submodule that in degree $m$ is generated by a lex-segment of length $H(M, m)$.

Moreover,

$$
H\left((F / L)_{\ell}, m\right)=H(F / M, m)_{\{m, r\}} \cdot
$$

Proof Assume that $M$ is a monomial submodule of $F$. Otherwise, let us take the generic initial module of $M$, gin $(M)$, with respect to the monomial order revlex. We get a new submodule which has the same Hilbert function of 
$M$ and moreover is a monomial submodule of $F$. We have that $\operatorname{gin}(M)_{x_{n}}=$ $\operatorname{gin}\left(M_{\ell}\right)$. If we work in generic coordinates, we can choose $\ell=x_{n}$ and so we get that $H\left((F / M)_{\ell}, d\right)=H\left((F / \operatorname{gin}(M))_{\ell}, d\right)$. So we may substitute $M$ by $\operatorname{gin}(M)$ in order to get a monomial submodule. Thus, we can write $M$ as $I_{1} e_{1} \oplus \cdots \oplus I_{r} e_{r}$, where each $I_{i}$ is a monomial ideal, hence a homogeneous ideal in $R$.

Let $d \in \mathbb{N}$ and $\ell$ be a generic linear form, then

$$
\begin{aligned}
& (F / M)_{\ell}=F /(M+\ell F) \cong F /\left[\left(I_{1}+(\ell)\right) e_{1} \oplus \cdots \oplus\left(I_{r}+(\ell)\right)\right] \cong \\
& \left(S / I_{1}\right)_{\ell} e_{1} \oplus \cdots \oplus\left(S / I_{r}\right)_{\ell} e_{r} .
\end{aligned}
$$

So we have that, by applying Green's HRT to each $\left(R / I_{i}\right)_{\ell}$,

$$
H\left((F / M)_{\ell}, m\right)=\sum_{i=1}^{r} H\left(\left(R / I_{i}\right)_{\ell}, m-f_{i}\right) \leq \sum_{i=1}^{r} H\left(R / I_{i}, m-f_{i}\right)_{\left\langle m-f_{i}\right\rangle} .
$$

Finally the last sum in (11) is bounded by $H\left((F / L)_{\ell}, m\right)$ where $L$ is a submodule such that $L_{m}$ is generated by a lex-segment of length $H(M, m)$ : this follows directly from Proposition 3.16.

\subsection{Green's HRT after scaling for modules}

We now derive from last theorem a result, that will give the possibility to apply Green's HRT in case it is difficult to have the Macaulay binomial representations. The idea is to consider the Hilbert function up to a multiplication by an integer. In this way, we will obtain a linear inequality, that will hold for all modules.

Corollary 3.19. Let $M$ be a module over $S=k\left[x_{1}, \ldots, x_{n}\right]$, generated in degree 0 , and let $\ell$ be a generic linear form, $d \in \mathbb{N}$, then

$$
H\left(M_{\ell}, d\right) \leq \frac{n-1}{n+d-1} H(M, d) .
$$

$\underline{\text { Proof }}$ For any integer $\delta$, we have that $H\left(\bigoplus_{i=1}^{\delta} M_{\ell}, d\right)=\delta H\left(M_{\ell}, d\right)$, so we can choose $\delta$ such that there is $\beta \in \mathbb{N}$ satisfying the following equality

$$
\delta H(M, d)=\beta \operatorname{dim}_{k}\left(S_{d}\right)=\beta\left(\begin{array}{c}
n+d-1 \\
d
\end{array}\right),
$$


so by Theorem 3.18 we have

$$
\begin{aligned}
& H\left(\bigoplus_{i=1}^{\delta} M_{\ell}, d\right) \leq \beta\left(\begin{array}{c}
n+d-1 \\
d
\end{array}\right)_{\langle d\rangle}=\beta\left(\begin{array}{c}
n+d-2 \\
d
\end{array}\right)= \\
& \beta\left(\begin{array}{c}
n+d-1 \\
d
\end{array}\right) \frac{n-1}{n+d-1}=\frac{n-1}{n+d-1} H\left(\bigoplus_{i=1}^{\delta} M, d\right),
\end{aligned}
$$

then, dividing by $\delta$, we get the claim.

Observation 3.20. This corollary can be described by the expression "after scaling" because the last fraction satisfies the following equality

$$
\frac{n-1}{n+d-1}=\frac{\operatorname{dim}\left(R_{d}\right)}{\operatorname{dim}\left(S_{d}\right)}
$$

where $S=k\left[x_{1}, x_{2}, \ldots, x_{n}\right]$ and $R=k\left[x_{1}, x_{2}, \ldots, x_{n-1}\right] \cong S_{\ell}$.

Therefore, the claim of the Corollary may be written as:

$$
\frac{H\left(M_{l}, d\right)}{H\left(S_{l}, d\right)} \leq \frac{H(M, d)}{H(S, d)}
$$

so we have scaled by respective value of the Hilbert function of the rings over which we have the two modules.

\section{Application to level algebras}

In this section we will apply the extension of the Green's HRT to level algebras.

Definition 4.1. Let $A=S / I=A_{0} \oplus \cdots \oplus A_{c}$ be an artinian $k$-algebra. Let us consider the socle of $A$, which is defined by $\operatorname{soc}(A)=\operatorname{ann}_{\mathbf{m}}(A)$, where $\mathbf{m}=\oplus_{i>0} A_{i}$. If $\operatorname{soc}(A)=\mathcal{U}_{0} \oplus \cdots \oplus \mathcal{U}_{c}$, we say that $A$ is level of type $b$ if $\operatorname{soc}(A)=\mathcal{U}_{c}=A_{c}$ and $\operatorname{dim}\left(A_{c}\right)=b$. We say that $A$ is Gorenstein when it is level of type 1 .

Let $A=A_{0} \oplus \cdots \oplus A_{c}$ be a level algebra with Hilbert function $\left(h_{0}, h_{1}, \ldots, h_{c}\right)$. By considering the dual of $A, A^{\vee}=\operatorname{Hom}_{k}(A, k)$, we get a level module, since 
is an artinian $S$-module with generators in a single degree, and with onedimensional socle, i.e. also concentrated in a single degree.

We want to consider the multiplication by a generic linear form $\ell \in A_{1}$.

We first apply Theorem 2.7. In this way we get the following bound on the Hilbert function of the ideal $(\ell)$.

$$
\operatorname{dim}_{k}(\ell)_{i} \geq h_{i}-\left(h_{i}\right)_{\langle i\rangle}, 0 \leq i \leq c .
$$

We can also apply Theorem 3.18 to the level module $M=A^{\vee}(-c)$. Notice that $M$ has Hilbert function $\left(h_{c}, h_{c-1}, \ldots, h_{0}\right)$. We get in this case the following bound for the module $\ell M$ :

$$
\operatorname{dim}(\ell M)_{i} \geq h_{c-i}-\left(h_{c-i}\right)_{\{i\}}, 0 \leq i \leq c .
$$

In the right hand side of this inequality, $a_{\{i\}}$ denotes $q\left(s_{i}\right)_{\langle i\rangle}+r_{\langle i\rangle}$, where $a=q s_{i}+r$ and $s_{i}=\left(\begin{array}{c}i+n-1 \\ i\end{array}\right)$.

We observe that $(\ell) \cong(\ell M)^{\vee}(-c+1)$. This implies that we can give two lower bounds for the Hilbert function of $(\ell)$, namely $h_{i}^{G M}$ and $h_{i}^{G}$, where we set $h_{i}^{G M}=h_{i}-\left(h_{i}\right)_{\{c-i\}}$ and $h_{i}^{G}=h_{i+1}-\left(h_{i+1}\right)_{\langle i+1\rangle}$.

We would like to find conditions in which $h_{i}^{G M}>h_{i}^{G}$, so when the bound given by the HRT for modules is better than the one given by the Green's HRT.

In [6], Geramita, Harima, Migliore and Shin, in case the polynomial ring $S$ has three variables, provided a list of all the possible Hilbert functions for level algebras of socle in degree 3,4,5 and in degree 6 and type 2. Among these, the condition $h_{i}^{G M}>h_{i}^{G}$ is true only for the ones contained in Table 1 in page 15.

Proposition 4.2. For $i$ such that the following conditions are all verified, we have that $h_{i}^{G M} \geq h_{i}^{G}$ :

$$
\left\{\begin{array}{l}
i-1 \leq c-i \\
h_{i}=h_{i+1} \\
s_{c-i}>h_{i+1}
\end{array}\right.
$$

$\underline{\text { Proof }}$ We have that $h_{i}^{G M}=h_{i}-\left(h_{i}\right)_{\{c-i\}}=h_{i+1}-\left(h_{i+1}\right)_{\{c-i\}}$. Since $\overline{s_{c-i}>} h_{i+1},\left(h_{i+1}\right)_{\{c-i\}}=\left(h_{i+1}\right)_{\langle c-i\rangle}$. We may then compare $\left(h_{i+1}\right)_{\langle c-i\rangle}$ and $\left(h_{i+1}\right)_{\langle i+1\rangle}$, using Lemma 3.11, and the fact that $i-1 \leq c-i$, so we get that 
$h_{i}^{G M} \geq h_{i}^{G}$

The previous condition does not characterize all cases in which $h_{i}^{G M}>h_{i}^{G}$, but only the ones in bold font in the table. Notice that if in the first column we have a certain number $i$ this means, according to our notation, that $h_{i-1}^{G M}>h_{i-1}^{G}$.

Question. What are the sufficient conditions for which the bound given by the HRT for modules is better than the bound given by the classical HRT?

\begin{tabular}{|c|c|c|c|}
\hline $\mathrm{Po}$ & $h$ & $h^{G M}$ & $h^{G}$ \\
\hline 2 & $1,3,3,3,2$ & $1,3,2,1$ & $1,2,3,2$ \\
\hline 2 & $1,3,3,3,3$ & $1,3,2,1$ & $1,2,3,3$ \\
\hline 2 & $1,3,3,3,3,2$ & $1,3,3,2,1$ & $1,2,3,3,2$ \\
\hline 4 & $1,3,6,8,5,2$ & $1,3,5,5,2$ & $1,3,6,4,2$ \\
\hline 4 & $1,3,6,9,5,2$ & $1,3,5,5,2$ & $1,3,6,4,2$ \\
\hline 4 & $1,3,6,10,6,2$ & $1,3,5,6,2$ & $1,3,6,5,2$ \\
\hline 2 & $1,3,3,3,3,3$ & $1,3,3,2,1$ & $1,2,3,3,3$ \\
\hline 4 & $1,3,6,8,5,3$ & $1,3,5,5,2$ & $1,3,6,4,3$ \\
\hline 4 & $1,3,6,10,6,3$ & $1,3,5,6,2$ & $1,3,6,5,3$ \\
\hline 2 & $1,3,3,3,3,3,2$ & $1,3,3,3,2,1$ & $1,2,3,3,3,2$ \\
\hline 3 & $1,3,4,4,4,3,2$ & $1,3,4,3,3,1$ & $1,3,3,4,3,2$ \\
\hline 3 & $1,3,4,4,4,4,2$ & $1,3,4,3,3,2$ & $1,3,3,4,4,2$ \\
\hline 4 & $1,3,5,6,5,4,2$ & $1,3,4,5,3,2$ & $1,3,5,4,4,2$ \\
\hline 4 & $1,3,6,8,6,4,2$ & $1,3,5,6,3,2$ & $1,3,6,5,4,2$ \\
\hline 5 & $1,3,6,8,10,6,2$ & $1,3,5,6,6,2$ & $1,3,6,8,5,2$ \\
\hline 5 & $1,3,6,9,10,6,2$ & $1,3,5,6,6,2$ & $1,3,6,8,5,2$ \\
\hline 5 & $1,3,6,9,11,6,2$ & $1,3,5,6,6,2$ & $1,3,6,9,5,2$ \\
\hline 5 & $1,3,6,9,12,6,2$ & $1,3,5,6,6,2$ & $1,3,6,9,5,2$ \\
\hline 5 & $1,3,6,10,10,6,2$ & $1,3,5,6,6,2$ & $1,3,6,8,5,2$ \\
\hline 5 & $1,3,6,10,11,6,2$ & $1,3,5,6,6,2$ & $1,3,6,9,5,2$ \\
\hline 5 & $1,3,6,10,12,6,2$ & $1,3,5,6,6,2$ & $1,3,6,9,5,2$ \\
\hline
\end{tabular}

Table 1: Hilbert functions of Level artinian algebras

Acknowledgments. I would like to thank my supervisor Mats Boij for his great support, and also Ralf Fröberg for the useful comments. Moreover, I 
also thank Giulio Caviglia for his suggestions, that were crucial in the last phase of preparation of the paper.

\section{References}

[1] J. Ahn And Y. S. Shin, Artinian level algebras of codimension 3, J. Pure Appl. Algebra, 216 (2012), pp. 95-107.

[2] M. BoiJ AND F. Zanello, Some algebraic consequences of Green's hyperplane restriction theorems, J. Pure Appl. Algebra, 214 (2010), pp. $1263-1270$.

[3] W. Bruns and J. Herzog, Cohen-Macaulay rings, vol. 39 of Cambridge Studies in Advanced Mathematics, Cambridge University Press, Cambridge, 1993.

[4] D. Eisenbud, Commutative algebra, vol. 150 of Graduate Texts in Mathematics, Springer-Verlag, New York, 1995. With a view toward algebraic geometry.

[5] V. Gasharov, Extremal properties of Hilbert functions, Illinois J. Math., 41 (1997), pp. 612-629.

[6] A. V. Geramita, T. Harima, J. C. Migliore, and Y. S. Shin, The Hilbert function of a level algebra, Mem. Amer. Math. Soc., 186 (2007), pp. vi+139.

[7] M. Green, Restrictions of linear series to hyperplanes, and some results of Macaulay and Gotzmann, in Algebraic curves and projective geometry (Trento, 1988), vol. 1389 of Lecture Notes in Math., Springer, Berlin, 1989, pp. $76-86$.

[8] H. A. Hulett, A generalization of Macaulay's theorem, Comm. Algebra, 23 (1995), pp. 1249-1263.

[9] G. Katona, A theorem of finite sets, in Theory of graphs (Proc. Colloq., Tihany, 1966), Academic Press, New York, 1968, pp. 187-207.

[10] M. Kreuzer and L. Robbiano, Computational commutative algebra. 2, Springer-Verlag, Berlin, 2005. 
[11] _ Computational commutative algebra 1, Springer-Verlag, Berlin, 2008. Corrected reprint of the 2000 original.

[12] J. B. KRuskaL, The number of simplices in a complex, in Mathematical optimization techniques, Univ. of California Press, Berkeley, Calif., 1963, pp. 251-278.

[13] F. S. Macaulay, The algebraic theory of modular systems, Cambridge Mathematical Library, Cambridge University Press, Cambridge, 1994. Revised reprint of the 1916 original, With an introduction by Paul Roberts.

[14] J. Migliore And F. Zanello, The strength of the weak Lefschetz property, Illinois J. Math., 52 (2008), pp. 1417-1433.

[15] K. Pardue, Nonstandard borel-fixed ideals, ProQuest LLC, Ann Arbor, MI, 1994. Thesis (Ph.D.)-Brandeis University.

Royal Institute of Technology, Department of Mathematics, S-10044 Stockholm, Sweden.

E-mail address: ogreco@kth.se 